\title{
3 Research Square \\ Identification and Verification of Candidate Genes Regulating Human Umbilical Vein Endothelial Cells Behavior Under Hyperglycemia
}

\section{XIAOYE MA}

Shanghai Tenth People's Hospital, Tongji University School of Medicine

\section{YUCHEN ZHOU}

Shanghai Tenth People's Hospital, Tongji University School of Medicine

JUNCHAO XIE

Shanghai Tenth People's Hospital, Tongji University School of Medicine

\section{GUILIN MENG}

Shanghai Tenth People's Hospital, Tongji University School of Medicine

YICHEN ZHAO

Shanghai Tenth People's Hospital, Tongji University School of Medicine

\section{YANXIN ZHAO}

Shanghai Tenth People's Hospital, Tongji University School of Medicine

Xueyuan Liu ( $\nabla$ lxyshsy@163.com )

Shanghai Tenth People's Hospital, Tongji University School of Medicine https://orcid.org/0000-00023869-8809

\section{Research article}

Keywords: Human umbilical vein endothelial cells, MicroRNA, Diabetes mellitus, Hyperglycemia,

Fibronectin

Posted Date: May 18th, 2020

DOI: https://doi.org/10.21203/rs.3.rs-26855/v1

License: (c) (i) This work is licensed under a Creative Commons Attribution 4.0 International License.

Read Full License 


\section{Abstract}

Background: Diabetes is a metabolic disease that has been widely demonstrated to be correlated with many microvascular and macrovascular diseases that seriously damage the patient's life quality. This study intended to investigate the underlying molecular mechanisms of endothelial dysfunction under hyperglycemia.

Methods: The gene expression profile of GSE49524 was downloaded and differentially expressed genes (DEGs) in hyperglycemia human umbilical cord endothelial cells (HUVECs) samples compared with normoglycemia HUVECs samples were identified by R software. Afterward, we analyzed the data by applying a combination of the bioinformatics method and used the microRNAs (miRNAs) databases to predict microRNAs that target key genes. The expression of the top 10 differentially expressed genes was validated through quantitative real-time PCR (qRT-PCR).

Results: A total of 65 genes were distinguished as DEGs. The dominant GO (gene ontology) terms and KEGG (Kyoto Encyclopedia of Genes and Genomes) pathways which were significantly overrepresented in the hyperglycemia HUVECs were identified. The results of the protein-protein interaction networks demonstrated that fibronectin 1 (FN1) is of the highest degree. In addition, several predicted miRNAs that target FN1 were obtained too. The further verification of the top 10 DEGs through qRT-PCR illustrated that nine of the up-regulated DEGs were up-regulated significantly in the hyperglycemia group when compared to the control group.

\section{Conclusions:}

This exploratory study may help to prompt an understanding of the underlying molecular mechanisms of the effect of hyperglycemia on the behavior of HUVECs and contribute to the production of effective therapeutic interventions.

\section{Background}

Diabetes is a metabolic disease that has been widely demonstrated to be correlated with many microvascular and macrovascular diseases that seriously damage the patient's life quality (1). In particular, hyperglycemia is associated with the disruption of endothelium's function, which could result in oxidative stress, the development of atherosclerosis, delayed-angiogenesis and the occurrence of cardiovascular and cerebrovascular events $(2,3)$. However, the molecular mechanisms underlying endothelial dysfunction in diabetic patients are still unknown, which limits efficient curative interventions in clinical researches.

Endothelial cells play important roles in vascular biology. They are not only functioning as blood vessels' protective lining, but also the local site that delivers oxygen to the tissue. The disruption of endothelium's function is thought to contribute to the development of macro-vascular diseases including atherosclerosis (4). The intactness of endothelial cell monolayer is of great importance for the regulation 
of local homeostasis and the protection of smooth muscle cells within the vessel from the harmful stimulus by providing a non-permeable barrier (5).

Human Umbilical Cord Endothelial Cells (HUVECs) obtained from mothers with gestational diabetes mellitus are an effective cellular model for early endothelial damage in diabetes (6). Therefore, by comparing the gene expression of hyperglycemic HUVECs with that of normoglycemia HUVECs in the Gene Expression Omnibus (GEO) database, we identified Differentially Expressed Genes (DEGs) and analyzed the Gene Ontology (GO) and Kyoto Encyclopedia of Genes and Genomes (KEGG) enrichment pathways in order to determine the key genes, pathways, and microRNAs (miRNAs) of HUVECs in highglucose state, thus revealing the potential molecular mechanism of high glucose on endothelial behaviors.

\section{Methods}

\subsection{Gene expression microarray data}

In this study, the gene expression profiles of GSE49524 were download from the NCBI-Gene Expression Omnibus (NCBI-GEO; http://www.ncbi.nlm.nih.gov/geo/). GSE49524 is based on the GPL7020 platform (NuGO array (human) NuGO_Hs1a520180). The GSE49524 dataset contained six samples, including three HUVEC samples from the umbilical cords of gestational diabetic mothers and three HUVEC samples from umbilical cord of controls (7). Umbilical cords were taken from three Caucasian women with Gestational diabetes (diagnosed not later than 28th gestational week [gw]) and from three Caucasian women without diabetes matching for age and Body Mass Index (BMI). All donors had normal blood pressure. A $100 \mathrm{~g} 3$ hours Oral Glucose Tolerance Test (OGTT) was conducted between the 24-34th gw.

\subsection{Identification of DEGs}

Quality control includes relative logarithmic expression and RNA degradation. In R software (Version 3.5.3, R Core Team, 2014), differentially expressed genes (DEGs) were identified through the Linear Models for Microarray Analysis(Limma) package (8). Limma provides a summary of the results of the linear model, performs hypothesis tests, and adjusts P-values for multiple testing, and the results include (log) fold changes, standard errors, t-statistics, and P-values. The Benjamini-Hochberg false discovery rate was used to correct the $P$ values. $P$ value $<0.05$ and $[\log F C]>1$ were used as the cut-off criteria of DEG analysis. A Volcano plot was carried out in R software using the GGPROT2 package $(9,10)$.

\subsection{GO enrichment and KEGG pathway analysis of the DEGs}

Gene Ontology(GO;www.geneontology.org) and Kyoto Encyclopedia of Genes and Genomes(KEGG;www.genome.ad.jp/KEGG) are widely used in bioinformatics identifying the most correlative molecular function(MF),biological process(BP), cellular component(CC) and relevant pathway 
information. In order to identify the significance of DEGs, KEGG and GO analysis were performed through the annotate package and clusterProfiler package in R software (11). P-value(adjusted by BenjaminiHochberg) $<0.05$ was used as the cut-off criteria.

\subsection{Construction of the PPI network of DEGs}

To further elucidate the molecular mechanism of hypoglycemia affecting the behavior of HUVEC cells, we made use of the Interactive Gene Retrieval Tool (STRING) database (http://www.string-db.org/) to evaluate the relationships among DEGs. Subsequently, we used Cytoscape software (Version 3.7.1, National Institute of General Medical Sciences) to analyze the PPI network.

\subsection{Cell culture}

Human umbilical vein endothelial cells (HUVECs) were purchased from Clonetics (San Diego, CA, USA) and were cultivated in DMEM low glucose medium supplemented with $2 \%$ fetal bovine serum with 100 $\mathrm{U} / \mathrm{mL}$ penicillin and $100 \mu \mathrm{g} / \mathrm{mL}$ streptomycin under standard culture conditions ( $\left.37^{\circ} \mathrm{C}, 5 \% \mathrm{CO} 2\right)$. HUVECs were treated with normal (NG, $5.5 \mathrm{mM})$ or high $(\mathrm{HG}, 25 \mathrm{mM})$ glucose for $24 \mathrm{~h}$, then collected for additional experiments.

\subsection{Quantitative PCR}

Cells were treated with Trizol reagent for extracting total RNA, and cDNA synthesis was performed using PrimeScript RT reagent kit (Takara Bio Inc., Otsu, Japan). The primers were designed and synthesized for the amplification of SIRT3, Mfn2 and $\beta$-actin by real-time quantitative PCR. The standard curve was obtained, and CT value was calculated. The mRNA expression of each gene was normalized to $\beta$-actin. The mixture (total volume $20 \mu \mathrm{l}$ ) used for PCR was as follows: DNA $(1 \mu \mathrm{l})$, SYBR FAST qPCR master mix $(10 \mu \mathrm{l})$, ROX High $(0.4 \mu \mathrm{l})$, Primer F $(10 \mathrm{pmol} / \mu \mathrm{l})(0.4 \mu \mathrm{l})$, and Primer R $(10 \mathrm{pmol} / \mu \mathrm{l})(0.4 \mu \mathrm{l})$. PCR was conducted as follows: $95^{\circ} \mathrm{C}$ for $3 \mathrm{~min}, 95^{\circ} \mathrm{C}$ for $3 \mathrm{sec}, 60^{\circ} \mathrm{C}$ for $30 \mathrm{sec}, 95^{\circ} \mathrm{C}$ for $15 \mathrm{sec}, 60^{\circ} \mathrm{C}$ for $15 \mathrm{sec}$, $95^{\circ} \mathrm{C}$ for $15 \mathrm{sec}$, for a total of 40 cycles.

\subsection{Prediction of miRNAs targeting the core gene}

MicroRNAs are a class of small non-coding RNAs that bind to the 3'-untranslated region of target mRNA to inhibit mRNA expression or the degradation of mRNAs (12). The key miRNAs targeting the core gene were predicted using TargetScan (http://www.targetscan.org/), miRDB (http:// www.mirdb.org/miRDB/index.html), and DIANA Tools (http://diana.imis.athenainnovation.gr/DianaTools/). 


\subsection{Statistical analysis}

Experiments were repeated at least three times. Data are expressed as means \pm standard error (SEM). Comparisons were made with two-tailed Student's t-test. $P<0.05$ was considered statistically significant.

\section{Results}

\subsection{The identification of differentially expressed genes}

We downloaded the gene expression profile GSE 49524 from GEO database, and DEGs were identified between hyperglycemia HUVECs samples and normoglycemia HUVECs samples using Limma package. We used $\mathrm{P}<0.05, \log \mathrm{FC}$ (fold control) $>1.0$ or $\log \mathrm{FC}<-1.0$ as the criteria, and 65 genes were identified as DEGs. A total of 65 genes were recognized as DEGs by applying $P<0.05, \log F C$ (fold control) $>1.0$ or $\operatorname{logFC}<-1.0$ as the criteria. Of these, the number of up-regulated genes was 51 , while the number of down-regulated genes was 14 . The volcano plot carried out in $\mathrm{R}$ software using the GGPROT2 package was also presented in Figure 1.

\subsection{GO term enrichment and KEGG pathway analysis}

Table 1 shows the top 10 enriched GO categories of the identified DEGs. The enrichment analysis of GO demonstrated that the predominant enriched GO terms belong to the biological process, such as extracellular structure organization, extracellular matrix organization, skeletal system development, and blood vessel morphogenesis and heart development. In addition to the biological process, there also existed some enriched $\mathrm{GO}$ terms associated with molecular function, such as cell adhesion molecule binding and growth factor binding. In addition, the DEGs were also enriched in GO terms related to cellular components, such as extracellular matrix, extracellular matrix component, endoplasmic reticulum lumen and proteinaceous extracellular matrix. Besides, $\mathrm{GO}$ terms in correlation with the cellular component group also enriched a few DEGs, such as extracellular matrix component, endoplasmic reticulum lumen, proteinaceous extracellular matrix and extracellular matrix. As shown in Figure 2, the results of KEGG analysis unveiled that the DEGs were enriched in PI3K-Akt signaling pathway, amoebiasis and AGE-RAGE signaling pathway in diabetic complications, and cellular senescence.

\subsection{DEGs' PPI network and hub genes in the PPI network}

The relevant information about DEGs and hub genes in PPI network are extracted from string database. A total of 21 nodes and 45 edges are contained in the PPI network. DEGs are indicated by the nodes, while the interactions between DEGs are symbolized by the edges. The top 10 high-degree hub nodes consisted of fibronectin 1(FN1), latent transforming growth factor beta binding protein 1(LTBP1), insulin like growth factor binding protein 3 (IGFBP3), collagen type III alpha 1 chain (COL3A1), collagen type IV alpha 1 chain(COL4A1), fibrillin 1 (FBN1), brain derived neurotrophic factor (BDNF), fibroblast growth factor 2 
(FGF2), transforming growth factor beta induced(TGFBI), transforming growth factor beta 2 (TGFB2). The first 10 high-degree core genes are composed of FN1 (fibronectin 1), LTBP1 (latent transforming growth factor beta binding protein 1), IGFBP3 (insulin like growth factor binding protein 3), COL3A1 (collagen type III alpha 1 chain), COL4A1 (collagen type IV alpha 1 chain), FBN1 (fibrillin 1), BDNF (brain derived neurotrophic factor), FGF2 (fibroblast growth factor 2), TGFBI (transforming growth factor beta induced), and TGFB2 (transforming growth factor beta 2). Of these genes, FN1 exhibited the highest nodal degree of 7 (Figure 3 ).

\subsection{Prediction of miRNAs that target the hub genes}

According to the information provided by the PPI network, we discovered that FN1 if of the highest degree and may be a key gene regarding the behavior of HUVECs under hyperglycemia. By using miRNA databases (TargetScan, miRDB, and DIANA Tools), we obtained the potential miRNAs targeting FN1 which include miR-429,miR-144-3p,miR-1-3p,miR-1271-5p,miR-206 and miR-96-5p (Figure 4). Besides, Figure 5 illustrated the binding sites between the predicted miRNAs and FN1.

\subsection{Validation of DEGs through qRT-PCR}

Table 2 lists the primer sequences applied in this study. Besides, the first 10 DEGs were selected for the validation experiments. The experimental results of qRT-PCR revealed that nine out of ten DEGs (FN1, LTBP1, IGFBP3, COL3A1, COL4A1, FBN1, BDNF, FGF2, TGFB2) were significantly up-regulated in the hyperglycemia group compared with the control group (Figure 6).

\section{Discussion}

In this study, we investigated the potential molecular mechanisms involved in vascular endothelial dysfunction in diabetes. The gene expression profile of GSE49524 was downloaded from GEO and raw data was analyzed by R software. A total of 65 DEGs were identified, composing of 14 under-expressed genes and 51 over-expressed genes. In addition, we further analyzed GO and KEGG pathways to prompt the understanding of the biological significance of the DEGs. The potential essential genes and miRNAs that may influence the behavior of HUVECs under hyperglycemia were identified through the combination of PPI networks and miRNA predictions.

The enrichment analysis of GO terms indicated that the DEGs were significantly enriched in extracellular matrix organization and extracellular structure organization. Furthermore, the results also demonstrated that the DEGs were correlated with blood vessel morphogenesis and heart development. Previous studies have revealed that angiogenesis plays an essential role in the impaired neovascularization in diabetes $(13,14)$. In addition, effective suppression of the damage of vascular endothelial cells or facilitation of angiogenesis may contribute to the effective treatment of diabetic vascular diseases $(15,16)$. 
Moreover, the DEGs were significantly enriched in several KEGG pathways, including ECM-receptor interaction, PI3K-Akt signaling pathway, AGE-RAGE signaling pathway in diabetic complications, TGFbeta signaling pathway, relaxin signaling pathway and FoxO signaling pathway. It has been demonstrated that AGE/RAGE signaling could enhance oxidative stress to facilitate the diabetesmediated calcification of vessels and result in the switch of VSMCs (vascular smooth muscle cells) phenotype to osteoblast-like cells phenotype (17). Previous studies have indicated that the exposure of HUVECs to high glucose may disrupt endothelial cells' functions such as insulin signaling (18) and the expression of a large number of proteins participating in the process of blood viscosity and thrombosis (19), which is consistent with our results. Therefore, understanding the role of the key pathways will allow for pharmacological intervention in the future.

Furthermore, the analysis of the PPI network unveiled that FN1, LTBP1,IGFBP3, COL3A1, COL4A1, FBN1, BDNF, FGF2, TGFBI, TGFB2 were the first 10 hub genes, which may be correlated with HUVECs behavior under hyperglycemia. The results of qRT-PCR demonstrated that the level of nine of the over-expressed DEGs (FN1, LTBP1, IGFBP3, COL3A1,COL4A1, FBN1, BDNF, FGF2,TGFB2) was higher in the hyperglycemia group than those in the control group. Of these genes, the node degree of FN1 is the highest. FN1 is a kind of extracellular matrix proteins and The expression of FN1 has been shown to be elevated significantly in Diabetic cardiomyopathy $(20,21)$. Accumulation of extracellular matrix proteins in diabetes leads to irreversible tissue damage (22), probably the result of tissue repair after cell death.

To further investigate the underlying molecular role of FN1, we used the miRNA databases (TargetScan, miRDB, and DIANA Tools) to predict the miRNAs that target FN1, and we identified the crossings between the results of the three databases, namely miR-429,miR-144-3p,miR-1-3p,miR-1271-5p,miR-206, and miR-96-5p. Afterward, we searched these six miRNAs in PubMed and noticed that four of them have been experimentally verified to target FN1, namely miR-429(23), miR-144-3p(24), miR-1271$5 p(25)$ and miR-206(26). Therefore, further experimental studies about miR-1-3p and miR-96-5p are needed to be confirmed.

There exist some limitations of this study. First, the sample sizes applied for expression spectrum analysis is small. Second, more detailed and in-depth research is required in the future. Hence, we anticipate that the results of this study can contribute to prompt a further understanding of the potential mechanisms that control the behavior of HUVECs under hyperglycemia.

\section{Conclusion}

In this study, we identified 65 genes as DEGs, among which 51 genes were up-regulated, and 14 genes were down-regulated. GO and KEGG pathway analyses were carried out to elucidate the molecular mechanisms of HUVECs under hyperglycemia. From the results of the PPI network, we found that FN1 had the highest degree and the predicted miRNAs targeting FN1 were obtained based on the miRNA databases (TargetScan, miRDB, and DIANA Tools), among which miR-429,miR-144-3p,miR-1$3 p, m i R-1271-5 p, m i R-206$ and $m i R-96-5 p$ were identified. 


\section{Abbreviations}

DEGs, differentially expressed genes; HUVECs, human umbilical cord endothelial cells; miRNAs, microRNAs; qRT-PCR, quantitative real-time PCR; FN1, Fibronectin 1; GEO, Gene Expression Omnibus; KEGG, Kyoto Encyclopedia of Genes and Genomes; BMI, Body Mass Index; OGTT, Oral Glucose Tolerance Test; LTBP1, Latent transforming growth factor beta binding protein 1; IGFBP3, Insulin like growth factor binding protein 3; COL3A1, Collagen type III alpha 1 chain; COL4A1, Collagen type IV alpha 1 chain; BDNF, Brain derived neurotrophic factor; FGF2, Fibroblast growth factor 2; TGFBI, Transforming growth factor beta induced; TGFB2, Transforming growth factor beta 2

\section{Declarations}

\section{Ethics approval and consent to participate}

Not applicable

\section{Consent for publication}

Not applicable

\section{Availability of data and materials}

The datasets used and analyzed during the current study are available in the NCBI-Gene Expression Omnibus (NCBI-GEO; https://www.ncbi.nlm.nih.gov/geo) online database.

\section{Competing interests}

The authors declare that they have no competing interests.

\section{Funding}

This work was supported by the National Natural Science Foundation of China [grant numbers 81771131] and Science and Technology Commission of Shanghai Municipality [grant numbers 17411950100,18140901900 and 19YF1438400].

\section{Authors's Contributions}

Yanxin Zhao and Xueyuan Liu designed the study; Junchao Xie, Yichen Zhao, Yuchen Zhou and Xiaoye Ma collected the data and carried out relevant experiments; Xiaoye Ma, Guilin Meng, Yuchen Zhou and 
Junchao Xie analyzed data and experimental results. Xiaoye Ma and Yuchen Zhou wrote the manuscript. Yanxin Zhao and Xueyuan Liu revised the paper. All authors read and approved the final manuscript.

\section{Acknowledgements}

Not applicable

\section{References}

1.Liu R, Luo Q, You W, Jin M. MicroRNA-106 attenuates hyperglycemia-induced vascular endothelial cell dysfunction by targeting HMGB1. Gene. 2018;677:142-8.

2.0'Keefe JH, Bell DS. Postprandial hyperglycemia/hyperlipidemia (postprandial dysmetabolism) is a cardiovascular risk factor. The American journal of cardiology. 2007;100(5):899-904.

3.Colette $\mathrm{C}$, Monnier L. Acute glucose fluctuations and chronic sustained hyperglycemia as risk factors for cardiovascular diseases in patients with type 2 diabetes. Hormone and metabolic research. 2007;39(09):683-6.

4.Piqueras L, Reynolds AR, Hodivala-Dilke KM, Alfranca An, Redondo JM, Hatae T, et al. Activation of $\mathrm{PPAR} \beta / \delta$ induces endothelial cell proliferation and angiogenesis. Arteriosclerosis, thrombosis, and vascular biology. 2007;27(1):63-9.

5.Mineo C, Deguchi H, Griffin JH, Shaul PW. Endothelial and antithrombotic actions of HDL. Circulation research. 2006;98(11):1352-64.

6.Di Tomo P, Lanuti P, Di Pietro N, Baldassarre MPA, Marchisio M, Pandolfi A, et al. Liraglutide mitigates TNF-alpha induced pro-atherogenic changes and microvesicle release in HUVEC from diabetic women. 2017;33(8).

7.Ambra R, Manca S, Palumbo MC, Leoni G, Natarelli L, De Marco A, et al. Transcriptome analysis of human primary endothelial cells (HUVEC) from umbilical cords of gestational diabetic mothers reveals candidate sites for an epigenetic modulation of specific gene expression. Genomics. 2014;103(56):337-48.

8.Ritchie ME, Phipson B, Wu D, Hu Y, Law CW, Shi W, et al. limma powers differential expression analyses for RNA-sequencing and microarray studies. Nucleic acids research. 2015;43(7):e47.

9.Li H, Zhao X, Wang J, Zong M, Yang H. Bioinformatics analysis of gene expression profile data to screen key genes involved in pulmonary sarcoidosis. Gene. 2017;596:98-104.

10.Ni M, Liu X, Wu J, Zhang D, Tian J, Wang T, et al. Identification of Candidate Biomarkers Correlated With the Pathogenesis and Prognosis of Non-small Cell Lung Cancer via Integrated Bioinformatics 
Analysis. Frontiers in genetics. 2018;9:469.

11.Tang L, Cheng Y, Zhu C, Yang C, Liu L, Zhang Y, et al. Integrative methylome and transcriptome analysis to dissect key biological pathways for psoriasis in Chinese Han population. Journal of dermatological science. 2018;91(3):285-91.

12.Wang J, Zhang C, He W, Gou X. Construction and comprehensive analysis of dysregulated long noncoding RNA-associated competing endogenous RNA network in clear cell renal cell carcinoma. 2018.

13.Wils J, Favre J, Bellien J. Modulating putative endothelial progenitor cells for the treatment of endothelial dysfunction and cardiovascular complications in diabetes. Pharmacology \& therapeutics. 2017;170:98-115.

14.Zhu ML, Yin YL, Ping S, Yu HY, Wan GR, Jian X, et al. Berberine promotes ischemia-induced angiogenesis in mice heart via upregulation of microRNA-29b. Clinical and experimental hypertension (New York, NY: 1993). 2017;39(7):672-9.

15.Gustafson D, Veitch S, Fish JE. Extracellular Vesicles as Protagonists of Diabetic Cardiovascular Pathology. Frontiers in cardiovascular medicine. 2017;4:71.

16.Targher G, Lonardo A, Byrne CD. Nonalcoholic fatty liver disease and chronic vascular complications of diabetes mellitus. Nature Reviews Endocrinology. 2018;14(2):99.

17.Kay AM, Simpson CL, Stewart JA. The role of AGE/RAGE signaling in diabetes-mediated vascular calcification. Journal of diabetes research. 2016;2016.

18.De Nigris V, Pujadas G, La Sala L, Testa R, Genovese S, Ceriello A. Short-term high glucose exposure impairs insulin signaling in endothelial cells. Cardiovascular diabetology. 2015;14:114.

19.Tabit CE, Chung WB, Hamburg NM, Vita JA. Endothelial dysfunction in diabetes mellitus: molecular mechanisms and clinical implications. Reviews in Endocrine and Metabolic Disorders. 2010;11(1):61-74.

20.Leask A. Potential therapeutic targets for cardiac fibrosis: TGFbeta, angiotensin, endothelin, CCN2, and PDGF, partners in fibroblast activation. Circulation research. 2010;106(11):1675-80.

21.Chen S, Puthanveetil P, Feng B, Matkovich SJ, Dorn GW, 2nd, Chakrabarti S. Cardiac miR-133a overexpression prevents early cardiac fibrosis in diabetes. Journal of cellular and molecular medicine. $2014 ; 18(3): 415-21$.

22.Falcao-Pires I, Leite-Moreira AF. Diabetic cardiomyopathy: understanding the molecular and cellular basis to progress in diagnosis and treatment. Heart failure reviews. 2012;17(3):325-44.

23.Engelsvold DH, Utheim TP, Olstad OK, Gonzalez P, Eidet JR, Lyberg T, et al. miRNA and mRNA expression profiling identifies members of the miR-200 family as potential regulators of epithelial- 
mesenchymal transition in pterygium. Experimental eye research. 2013;115:189-98.

24.Liang W, Xie Z, Cui W, Guo Y, Xu L, Wu J, et al. Comprehensive gene and microRNA expression profiling reveals a role for miRNAs in the oncogenic roles of SphK1 in papillary thyroid cancer. Journal of cancer research and clinical oncology. 2017;143(4):601-11.

25.Gong J, Wang ZX, Liu ZY. miRNA1271 inhibits cell proliferation in neuroglioma by targeting fibronectin 1. Molecular medicine reports. 2017;16(1):143-50.

26.Duan J, Zhang X, Zhang S, Hua S, Feng Z. miR-206 inhibits FN1 expression and proliferation and promotes apoptosis of rat type II alveolar epithelial cells. Experimental and therapeutic medicine. 2017;13(6):3203-8.

\section{Figures}




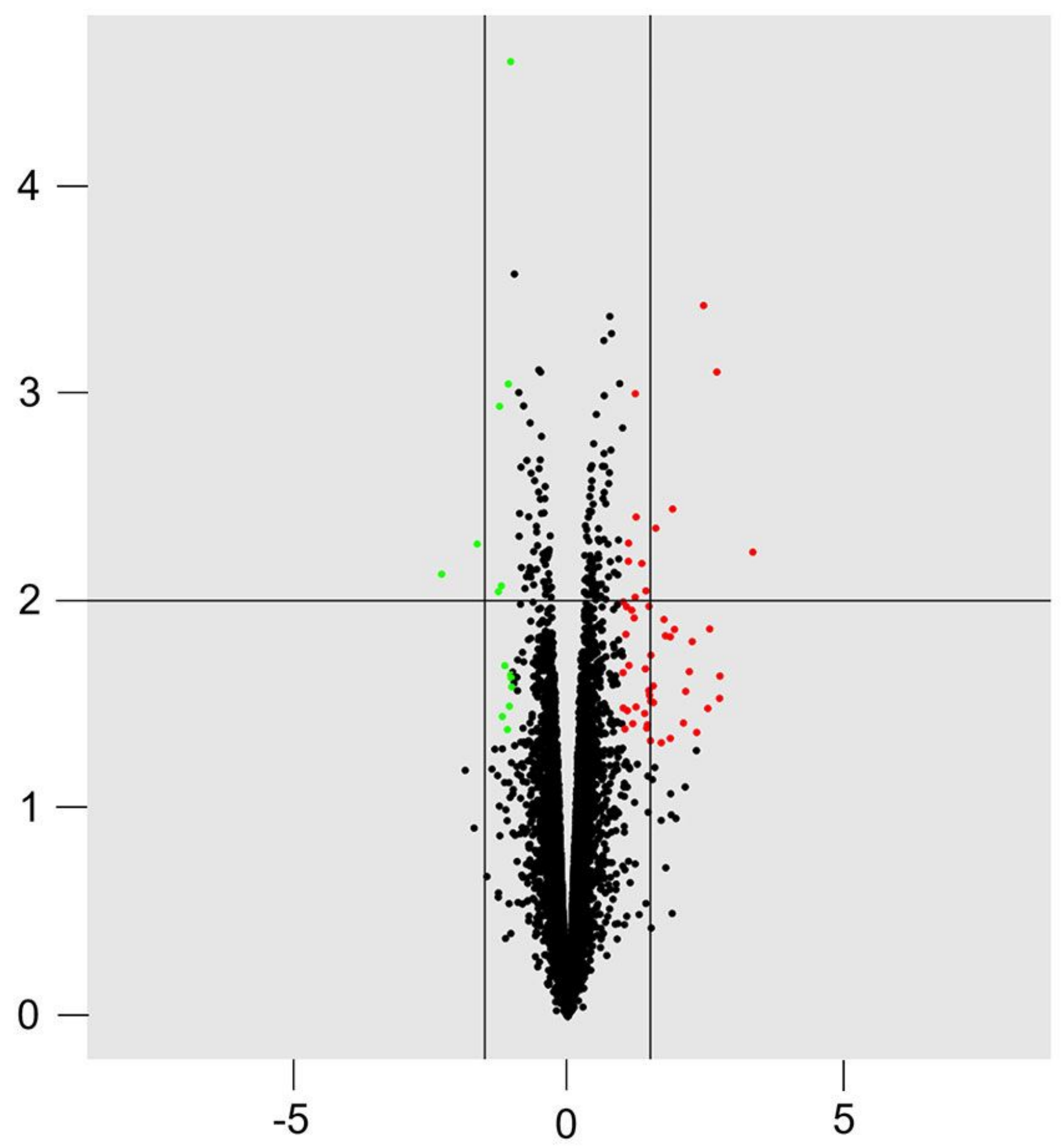

Figure 1

The volcano plot carried out in R software using GGPROT2 package. One dot represents one gene. Red, up-regulated; blue, down-regulated; black, no difference 


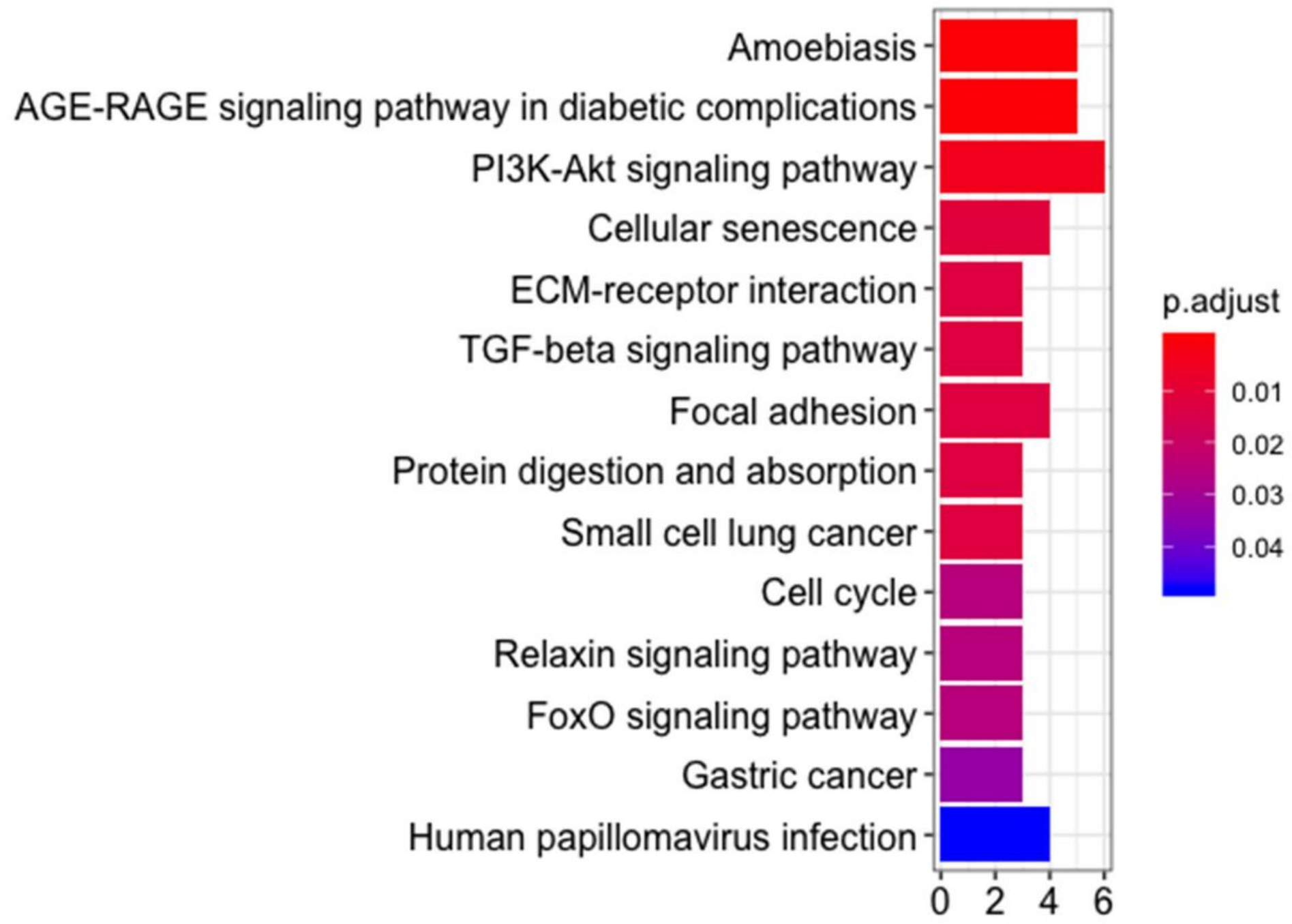

Figure 2

KEGG pathway analysis. The significant enriched Kyoto Encyclopedia of Genes and Genomes pathways terms of DEGs 


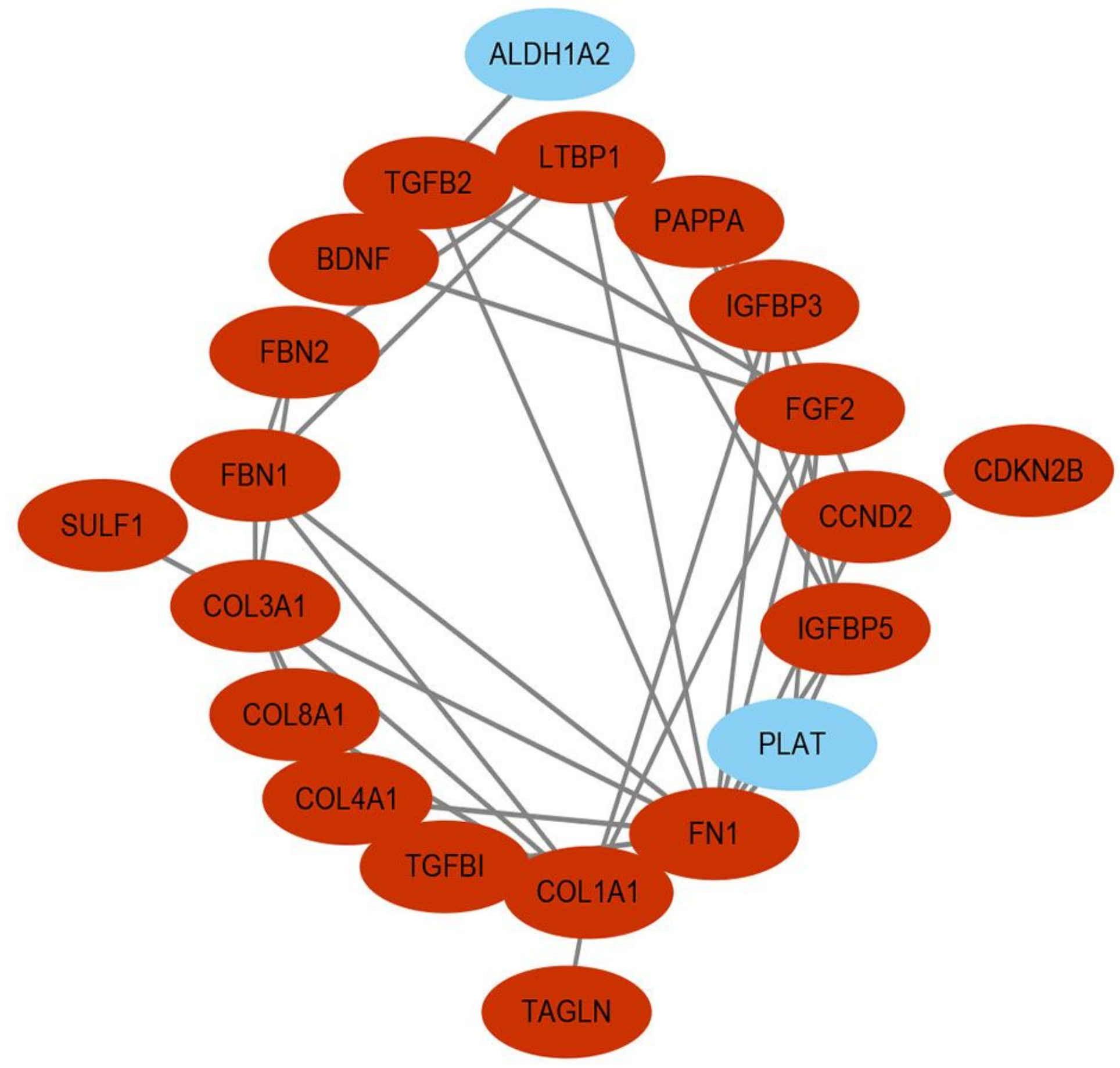

Figure 3

PPI network. PPI network unveiled that FN1, LTBP1,IGFBP3, COL3A1, COL4A1, FBN1, BDNF, FGF2, TGFBI, TGFB2 were the first 10 hub genes. Red, up-regulated; blue, down-regulated 


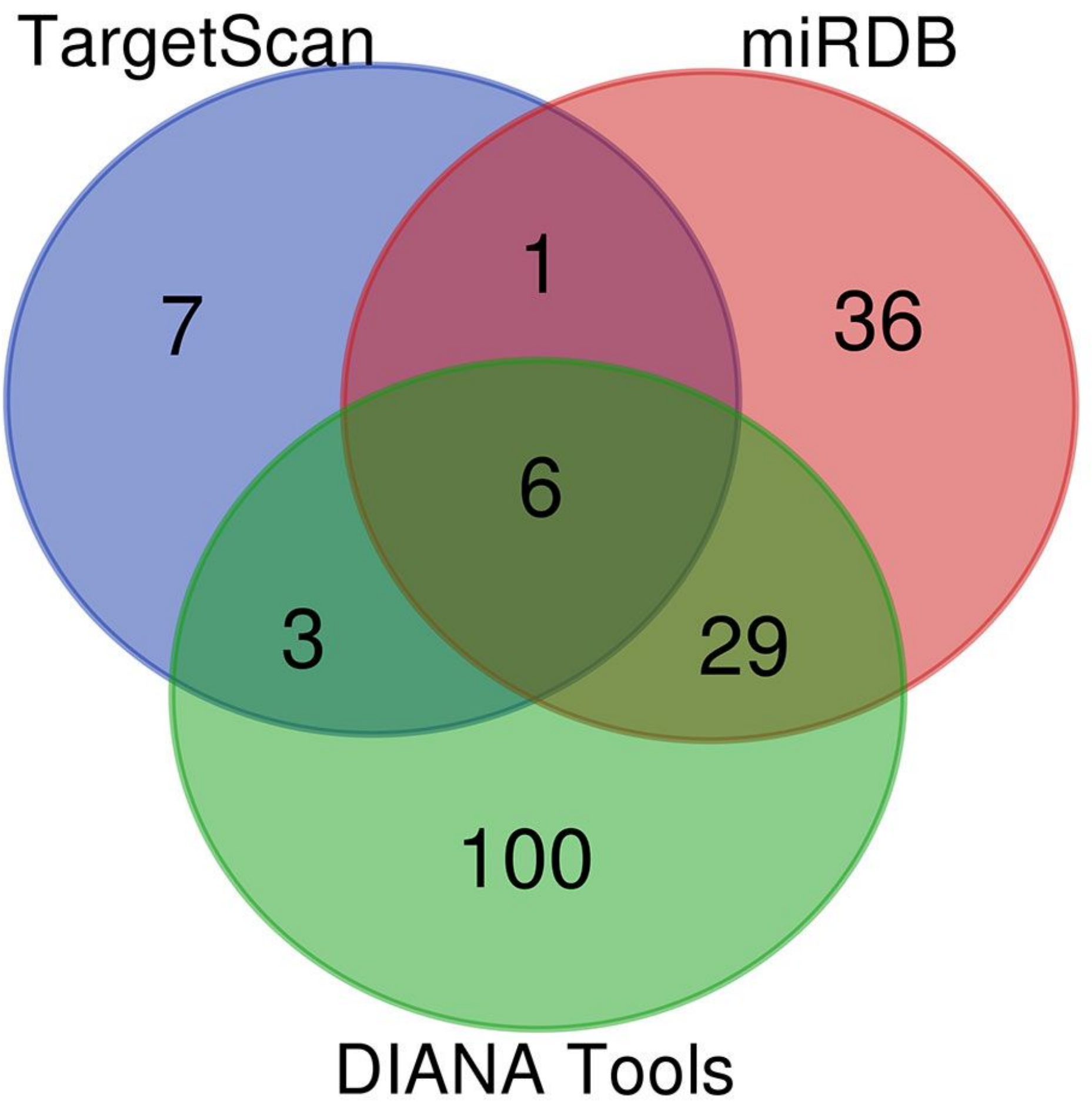

Figure 4

Identification of miRNAs targeting FN1 using the databases of miRNAs (TargetScan, miRDB, and DIANA Tools). 
Position 1249-1256 of FN1 3' UTR 5' ${ }^{\prime}$. . UUUAAUAaAaGaUUUACAUUCCA...

hsa-miR-1-3p

$3^{\prime}$ UAUGUAUGAGGAAAUGUAGGU

Position 811-817 of FN1 3' UTR 5' . . GUAUUCAAUACCGCUCAGUAUUU... .

hsa-miR-429

3' UGCCAAAAUGGUCUGUCAUAAU

Position 683-690 of FN1 $3^{\prime}$ UTR $5^{\prime}$. . UUUUAAUAAAAGAUUUACAUUCCA.

hsa-miR-206

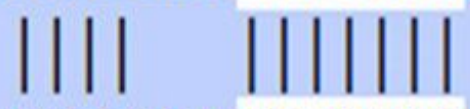

$1|1| 1 \mid$

3' GGUGUGUGAAGGAAUGUAAGGU

Position $1199-1206$ of FN1 $3^{\prime}$ UTR $5{ }^{\prime}$ ' ...UUUUUUUAUACUGUAUGUGCCAAA...

hsa-miR-1271-5p

3' ACUCACGAACGAUCCACGGUUC

Position 1190-1197 of FN1 3' UTR 5' . . GGGUGAUUAUUUUUUUAUACUGUA...

hsa-miR-144-3p

3' UCAUGUAGUAGAUAUGACAU

Position 1199-1206 of FN1 $3^{\prime}$ UTR $5{ }^{\prime}$. . . UUUUUUUAUACUGUAU--GUGCCAAA...

hsa-miR-96-5p

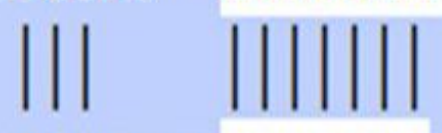

3' UCGUUUUUACACGAUCACGGUUU

Figure 5

The putative binding sites of the predicted miRNAs in the 3'-UTR of FN1. 


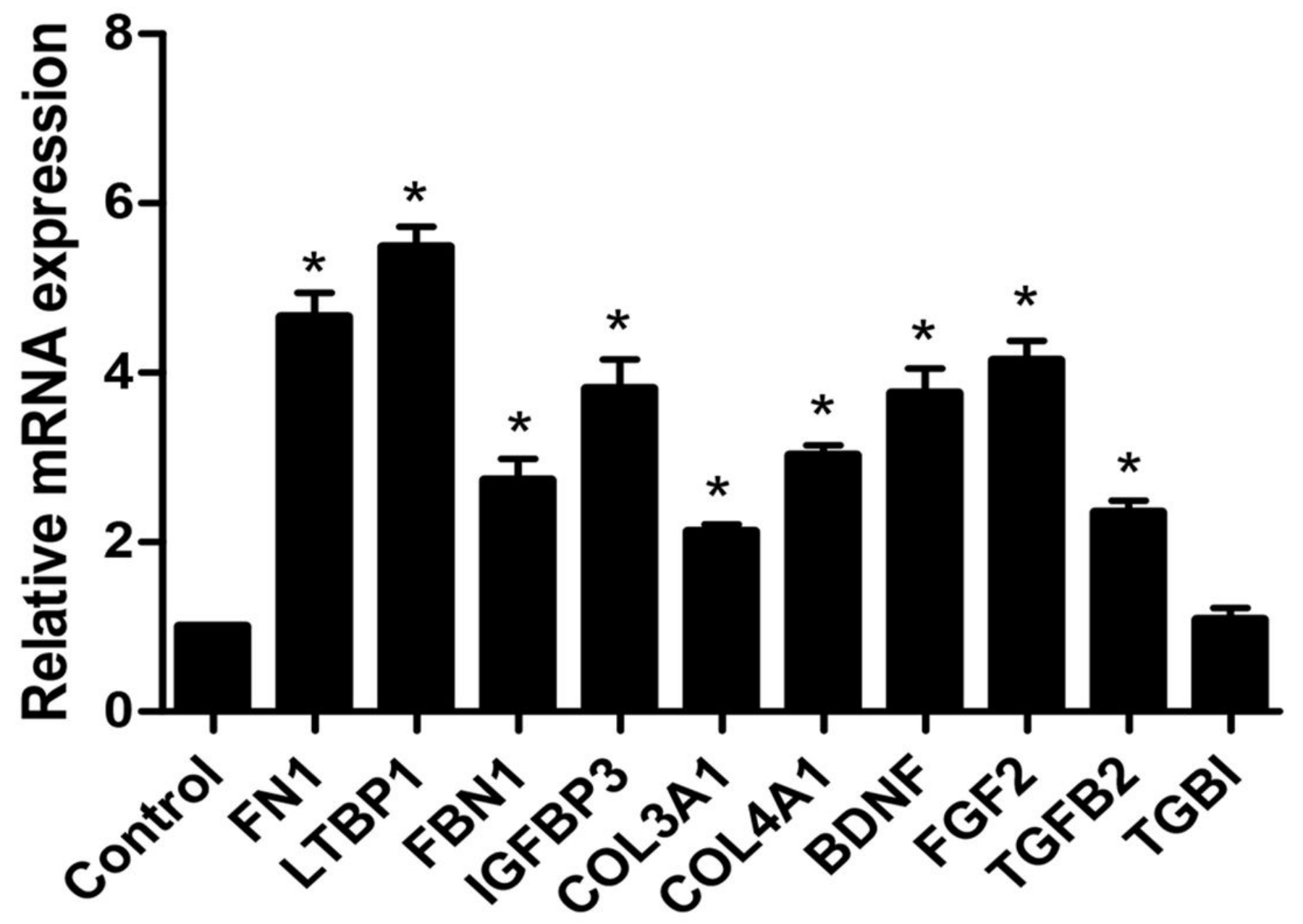

Figure 6

qRT-PCR validation of the top ten DEGs. ( $\left.{ }^{*}<<0.05 ; n=3\right)$ 